\title{
Prognostic Factors for Persistence of Chronic Abdominal Pain in Children
}

\author{
${ }^{*}$ Marieke J. Gieteling, *Sita M.A. Bierma-Zeinstra, ${ }^{\ddagger} Y$ vonne Lisman-van Leeuwen, \\ ${ }^{\dagger}$ Jan Passchier, and ${ }^{\ddagger}$ Marjolein Y. Berger
}

\begin{abstract}
Objectives: The aim of the study was to identify prognostic factors for the persistence of chronic abdominal pain (CAP) in children.

Materials and Methods: For this systematic review, MEDLINE, EMBASE, and PsycINFO were searched up to June 2008 for prospective follow-up studies of pediatric CAP as defined by the criteria of Apley, von Baeyer, or the Rome Committee. The outcome measure of interest was persistence of CAP. Persistent CAP was considered only when the abdominal pain of children with CAP persisted during follow-up at the same level of frequency and severity. For each study the risk for bias was assessed. The evidence for prognostic factors was summarized according to a best-evidence synthesis.

Results: Eight studies, which examined 17 prognostic factors, were included. Moderate evidence was found that having a parent with gastrointestinal symptoms predicts the persistence of CAP. Strong evidence was found for no association between female sex and the duration of CAP, and moderate evidence that the severity of abdominal pain does not predict persistence of CAP. There is conflicting evidence as to whether psychological factors prevent, or have no relation to, persistence of CAP.

Conclusions: Because there are few prognostic follow-up studies on pediatric CAP, the evidence for prognostic factors is limited. Physicians should ask about parental gastrointestinal problems because this is a risk factor for persistence of CAP in children. The hypothesis that psychological factors of the child predict persistence of CAP is not supported by evidence from follow-up studies.
\end{abstract}

Key Words: children, chronic abdominal pain, functional gastrointestinal disorders, prognosis, recurrent abdominal pain, risk factor

(JPGN 2011;52: 154-161)

Received April 27, 2009; accepted May 5, 2010.

From the *Department of General Practice, the tDepartment of Medical Psychology and Psychotherapy, Erasmus Medical Center, University Medical Center, Rotterdam, and the $\ddagger$ Department of General Practice, University Medical Center Groningen, University of Groningen, The Netherlands.

Address correspondence and reprint requests to Marjolein Y. Berger, PhD, General Practitioner, Department of General Practice, University Medical Center Groningen, University of Groningen, PO Box 196, 9700 AD Groningen, The Netherlands (e-mail: m.y.berger@med. umcg.nl).

Supplemental digital content is available for this article. Direct URL citations appear in the printed text, and links to the digital files are provided in the HTML text of this article on the journal's Web site (www.jpgn.org).

The authors report no conflicts of interest.

Copyright (C) 2011 by European Society for Pediatric Gastroenterology, Hepatology, and Nutrition and North American Society for Pediatric Gastroenterology, Hepatology, and Nutrition

DOI: 10.1097/MPG.0b013e3181e82a28 $\longrightarrow$ hronic abdominal pain (CAP) or recurrent abdominal pain (RAP) is a prevalent disorder that has major implications for a child's well-being and the health care system (1). CAP was first described by Apley and Naish (2) as abdominal pain that occurred in at least 3 episodes in at least 3 months and was severe enough to affect a child's activities. Apley and Naish (2) postulated that CAP was a functional syndrome that could not be explained by organic pathology; however, this assumption was difficult to establish using their definition. Therefore, to reduce misclassification between functional and organic abdominal pain, the definition of CAP has been revised during the years $(3,4)$. von Baeyer and Walker (4) suggested studying children who fulfilled criteria for RAP and described the tests performed to preclude organic disease to become informed about the magnitude of the role of organic disease. To define functional abdominal pain as a positive diagnosis rather than a diagnosis per exclusionem, the Rome Committee described symptom-based diagnostic criteria and reported the minimum examinations needed to exclude organic pathology (3).

CAP is proposed as an altered pain sensation due to a dysfunction of the brain-gut axis resulting from a complex interaction among biological, psychological, and social factors. The gut and the brain are highly integrated and communicate in a bidirectional fashion. Emotion, behavior, gut function, and pain are interrelated in this model of thinking (5). Children with CAP have more anxiety disorders, depressive symptoms, and other somatic complaints, and have experienced more negative life events than unaffected children (6). Parents of children with CAP have more gastrointestinal (GI), anxiety, and depressive symptoms than control parents (7).

Despite treatment, approximately $30 \%$ of children with CAP have long-lasting complaints, and there is evidence that CAP is a risk factor for the occurrence of irritable bowel syndrome in adulthood $(8,9)$. Psychosocial factors of the child are reported to be associated with the prognosis of CAP (10). Evidence in favor of such a relation comes mainly from case-control studies; however, such a retrospective study design cannot elucidate whether associated factors cause the pain, influence the course of the pain, or are a result of the pain. Insight into the potential prognostic factors may help clinicians to recognize a child at risk for persistent abdominal pain and guide clinical management.

Although knowledge on factors influencing the clinical course of CAP is important for physicians and patients with regard to patient information, anticipation of treatment possibilities, and identification of children at high risk, no overview of these prognostic factors is currently available. Good-quality prognostic studies and systematic reviews are the basis of evidence-based guidelines. Therefore, the aim of the present review was to systematically describe, investigate, and summarize the quantity and quality of all of the current evidence for potential prognostic factors for the persistence of CAP in children. 


\section{MATERIALS AND METHODS}

A search was made of the MEDLINE database of articles from 1965 to June 2008, in EMBASE from 1980 to June 2008, and in PsycINFO from 1970 to June 2008. The key words used to describe the study population were "abdominal pain," "chronic abdominal pain," "recurrent abdominal pain," and "functional abdominal pain." To detect the relevant age group the terms "child," "infant," and "adolescent" were used. Search strategies with a high sensitivity for follow-up studies, as recommended by Altman (11) (MEDLINE) and Wilczynski and Haynes (12) (EMBASE), were used. No language restriction was applied. Additional strategies to identify studies included hand searching the reference lists of the follow-up studies on pediatric CAP of recently published review articles and of articles written by experts in the field.

Two reviewers (M.J.G. and M.Y.B.) independently screened all of the abstracts of the identified articles for eligibility. Full articles were retrieved if the abstract provided insufficient information to enable selection, or if the article had passed the first eligibility screening. An article was eligible if it met all of the following criteria:

1. The study population concerned children or adolescents ages 4 to 18 years.

2. CAP was described using the criteria defined by Apley and Naish (2) (Introduction), von Baeyer and Walker (4) (2-stage classification for RAP: stage 1: abdominal pain that occurs at least once each month, in at least 3 consecutive months, within the last year, and these episodes are usually severe enough to stay home from school, terminate or avoid play, or take medication for the pain; and the child rates the pain as moderate to severe; stage 2: RAP subdivided in either functional or organic disease based on a predefined medical evaluation (4)), or the Rome Committee (at least 12 weeks of nearly continuous abdominal pain in school-age children, no or only occasional relation of pain with physiological events, some loss of daily function, the pain is not feigned, insufficient criteria for other functional GI disorder that would explain the abdominal pain, and physical examinations, growth, laboratory test, and abdominal ultrasound should be normal) (3).

3. The study determined prognostic factors for persistence of chronic abdominal pain in children with CAP.

4. The frequency and duration of the chronic abdominal pain at follow-up were comparable to those of the CAP at the start of the study.

5. The outcome was determined prospectively.

There were no restrictions on duration of follow-up. Any disagreement regarding inclusion was resolved by consensus or by arbitration of a third reviewer (S.M.A.B.-Z.).

Two reviewers (M.J.G. and S.M.A.B.-Z.) independently assessed the risk for bias in each study. The instrument used was a modified version of an established criteria list used in systematic reviews of prognostic factors $(13,14)$. We modified the criteria list based on the framework for assessing internal validity of studies of prognosis as described by Altman (11), Hayden et al (15), and the STROBE statement (16). The final list consisted of 16 items, each having a "yes"/"no"/"don't know" answer option: a "yes" was scored as 1, "no" and "don't know" were scored as zero (range 016) (Supplemental Digital Content 1 at http://links.lww.com/MPG/ A21). Studies at low risk for bias had high final scores. The interassessor agreement on the risk for bias was calculated using

\begin{tabular}{lc}
\hline TABLE 1. Levels of evidence for prognostic factors \\
\hline Strong evidence & $\begin{array}{c}\text { Consistent findings }(>75 \%) \text { in at } \\
\text { least } 2 \text { LRB cohorts } \\
\text { Consistent findings }(>75 \%) \text { in } 1 \mathrm{LRB} \\
\text { cohort and at least } 1 \mathrm{HRB} \text { cohort }\end{array}$ \\
Moderate evidence & $\begin{array}{l}\text { Findings of } 1 \mathrm{LRB} \text { cohort or consistent } \\
\text { findings }(>75 \%) \text { in at least } \geq 3 \mathrm{HRB}\end{array}$ \\
Weak evidence & cohorts \\
Insufficient & $<3$ HRB cohorts available \\
Conflicting & $\begin{array}{l}\text { Inconsistent findings irrespective of the } \\
\text { risk for bias }\end{array}$ \\
No evidence & No data presented
\end{tabular}

$\mathrm{HRB}=$ high-risk for bias; $\mathrm{LRB}=$ low-risk for bias; $\mathrm{RB}=$ risk for bias.

kappa scores $(>0.7=$ a high level of agreement between assessors; $0.5-0.7=\mathrm{a}$ moderate level of agreement, and $<0.5=$ a poor level of agreement).

Data extraction was performed by M.J.G. and verified by a second author (M.Y.B.). Extracted information included patient characteristics, the study setting, follow-up period, numbers lost to follow-up, prognostic factors, and measures of associations.

Because the studies were heterogeneous with respect to definition of the study population, study setting, duration of follow-up, and the prognostic factors studied, we refrained from metaanalysis (because pooling would give incorrect estimates of the real effects) and instead performed a best-evidence synthesis.

The levels of evidence for the associations found are based on the guidelines of Sackett et al (17) and are presented in Table 1. Studies were defined as being at low risk for bias when they scored more than $55 \%$ of the maximum score, that is, $\geq 9$.

Our outcome measure of prognosis of CAP was defined as the percentage of patients with persisting CAP after follow-up. Where possible, the measure of association of a prognostic variable with the percentage of children with persistent CAP after follow-up is presented as relative risk (RR) or an odds ratio (in case of a logistic regression model), with corresponding 95\% confidence interval and $P$ value (Fisher exact test). If the authors only provided the direction of the association and the statistical significance, then this was presented. A $P \leq 0.05$ was considered significant. Prognostic variables with significant RRs or odds ratios were considered to be associated. To take into account that smaller studies are less likely to find significant associations, in studies with a sample size $<50$, we also considered RRs $\leq 0.5$ and RRs $\geq 2$ to be related.

\section{RESULTS}

\section{Search Results}

A total of 2968 potentially relevant articles were identified on the first search. Most articles were discarded after analysing the abstracts because they were not follow-up studies. Of the 32 followup studies that remained, 14 studies were excluded: 2 because they studied the incidence of CAP $(18,19), 4$ because the definition of CAP did not fulfill our criteria $(20-23)$ (the abdominal pain at baseline or follow-up was of shorter duration or of less frequency), and 8 because their outcome measures were different from ours (24-31). The outcome measures presented in these excluded studies were health-related functional disability $(26,28,30,31)$, severity of abdominal pain (27), a level of somatization defined as a score on a somatization index scale $(24-26,28-30)$, or coexisting symptoms after follow-up (31). Of the remaining 
18 follow-up studies, 10 were excluded because they did not study prognostic factors $(32-41)$. Consequently, 8 studies were included in the final review (42-49).

\section{Assessment of Risk for Bias}

Details on the assessment of potential biases of the included studies are presented in Table 2 . The interobserver reliability of this assessment (kappa 0.75) was good. Using our cutoff point of $\geq 9,5$ studies (42-46) were classified as studies with a low risk for bias.

The most important methodological shortcomings concerned the following items. Most studies did not describe the study population in sufficient detail; for example, in most of the studies, referral status, prior investigations, relevant comorbidity (items B, C), and the moment in the course of the disease (item A) were not clear. Only 2 studies compared the patient and pain characteristics of those lost to follow-up with those who were followed (item G) $(44,48)$. Two studies reported on treatment of the patients during follow-up (item $H)(45,49)$. In addition, in the statistical analysis, 1 study adjusted for age, sex, and baseline severity of abdominal pain (item P) (44).

\section{Study Characteristics}

The study characteristics and the prognostic factors are presented in Table 3 . The sample size of the included studies ranged from 22 (48) to 153 (47). The percentage of children with persistent CAP ranged from $28.9 \%$ (42) to $52.9 \%$ (49). Six studies used the criteria of Apley and Naish (2), but all of these excluded children with organic pathology by medical testing $(43,45-49)$, and 2 studies used the Rome II criteria $(42,44)$. Five studies $(44-48)$ were conducted in tertiary care (pediatric gastroenterology) and 3 in secondary care (pediatrics) $(42,43,49)$. Four studies $(44,45,48,49)$ investigated inpatients and 3 investigated outpatients $(43,46,47)$. In 1 article (42) it was unclear whether the study concerned inpatients or outpatients.

\section{Best-evidence Synthesis}

The results of the best-evidence synthesis are presented in Table 4. Seventeen potential prognostic factors for the persistence of CAP were identified, of which 10 were studied in 1 study only.

Female sex was studied in 6 studies $(42-44,46,48,49)$, of which 4 had a low risk for bias $(42-44,46,50)$. Of these 4 latter studies, in 1 study female sex predicted the persistence of CAP (43), whereas in the remaining 3 , sex was not associated with the outcome. The best-evidence synthesis showed strong evidence that female sex and persistence of CAP are not related.

Four psychological factors were studied in 4 studies (43$45,47)$, of which 3 had a low risk for bias $(43-45)$. There was weak evidence that behavioral disturbances and psychological disorders do not predict persistence of CAP. There was conflicting and insufficient evidence that depressive/anxiety disorders and academic competence, respectively, predict persistence of CAP. Altogether, we found conflicting evidence as to whether "any", psychological factor of the child prevents or has no predictive value for persistence of CAP.

The severity of baseline abdominal pain was examined in 3 studies $(44,47,48)$, of which 1 had a low risk for bias (44). In all 3 studies, there was no association between the severity of the baseline CAP and persistence of CAP. Consequently, there was moderate evidence that the severity of abdominal pain at baseline does not predict the persistence of CAP.

The effect of negative life events on the persistence of CAP was investigated in only 1 study (44) with low risk for bias. In that study, more children with high levels of negative life events at baseline had persistence of CAP. Therefore, there was weak evidence that negative life events predict the persistence of CAP.

The effect of having a parent with functional GI symptoms on the persistence of CAP was examined in 2 studies $(45,48)$. One study (45) showed low risk for bias. In both studies, having a parent with GI symptoms was associated with a higher risk for the persistence of CAP. There was moderate evidence that these factors predict the persistence of CAP in children.

One study, which showed low risk for bias, investigated the association between the perception of parents of the illness of their child and the prognosis of CAP (44). It was found that having parents who continue to search for an organic explanation of the pain (expressed by a high number of consultants) and parents who refused to consider a psychological influence on the pain (expressed by disagreement with psychological referral) was associated with the persistence of CAP. Therefore, there was weak evidence that parental perception of illness predicts the persistence of CAP in their children.

Because of the small number of studies or conflicting directions of the associations, it was not clear whether age, educational level, duration of CAP at baseline, presence of associated symptoms, a history of 2 or more surgical operations, and/or socioeconomic status of the family influenced the course of pediatric CAP.

\begin{tabular}{|c|c|c|c|c|c|c|c|c|c|c|c|c|c|c|c|c|c|c|}
\hline Item & A & B & $\mathrm{C}$ & $\mathrm{D}$ & E & $\mathrm{F}$ & G & $\mathrm{H}$ & I & $\mathrm{J}$ & $\mathrm{K}$ & $\mathrm{L}$ & $\mathrm{M}$ & $\mathrm{N}$ & $\mathrm{O}$ & $\mathrm{P}$ & Total & $\mathrm{RB}$ \\
\hline \multicolumn{19}{|l|}{ References } \\
\hline Pace et al (42) & 0 & 1 & 0 & 1 & 1 & 1 & 0 & 0 & 1 & 1 & 0 & 1 & 1 & 0 & 1 & 0 & 9 & LRB \\
\hline Stordal et al (43) & 0 & 1 & 0 & 1 & 1 & 1 & 0 & 0 & 1 & 1 & 1 & 1 & 1 & 0 & 1 & 0 & 10 & LRB \\
\hline Lindley et al (44) & 0 & 1 & 1 & 0 & 1 & 1 & 1 & 0 & 0 & 0 & 0 & 0 & 1 & 1 & 1 & 1 & 9 & LRB \\
\hline Crushell et al (45) & 0 & 0 & 1 & 0 & 1 & 1 & 0 & 1 & 0 & 0 & 1 & 1 & 1 & 1 & 1 & 0 & 9 & LRB \\
\hline Walker et al (46) & 0 & 0 & 0 & 1 & 1 & 1 & 0 & 0 & 1 & 0 & 1 & 1 & 1 & 1 & 1 & 0 & 9 & LRB \\
\hline Walker and Heflinger (47) & 0 & 1 & 0 & 1 & 1 & 1 & 0 & 0 & 1 & 0 & 1 & 1 & 1 & 0 & 0 & 0 & 8 & HRB \\
\hline Magni et al (48) & 0 & 0 & 0 & 0 & 1 & 0 & 1 & 0 & 0 & 0 & 1 & 0 & 1 & 0 & 1 & 0 & 3 & HRB \\
\hline Christensen and Mortensen (49) & 0 & 0 & 0 & 0 & 1 & 1 & 0 & 1 & 1 & 1 & 0 & 1 & 1 & 0 & 1 & 0 & 8 & HRB \\
\hline Total score & 0 & 4 & 2 & 4 & 8 & 7 & 2 & 2 & 5 & 3 & 5 & 6 & 8 & 3 & 7 & 1 & & \\
\hline
\end{tabular}

The letters A to P correspond with the 16 criteria mentioned in Supplement 1, http://links.lww.com/MPG/A21, each criteria could be scored as 0 or 1 ("yes", was scored as 1; "no" and "don't know" were scored as 0 ). $\mathrm{HRB}=$ high-risk for bias; $\mathrm{LRB}=$ low-risk for bias; $\mathrm{RB}=$ risk for bias. 


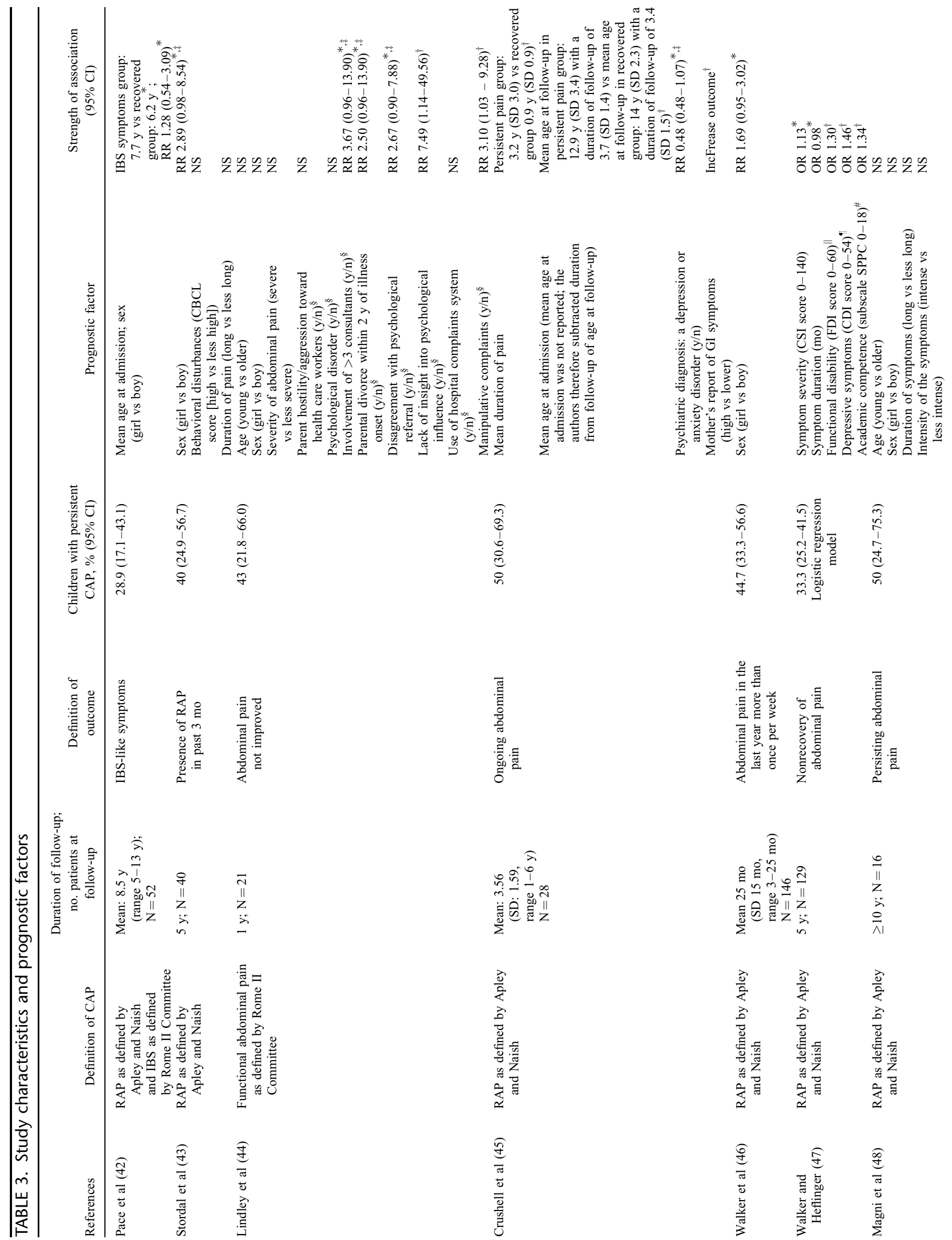

www.jpgn.org

Copyright 2011 by ESPGHAN and NASPGHAN. Unauthorized reproduction of this article is prohibited. 


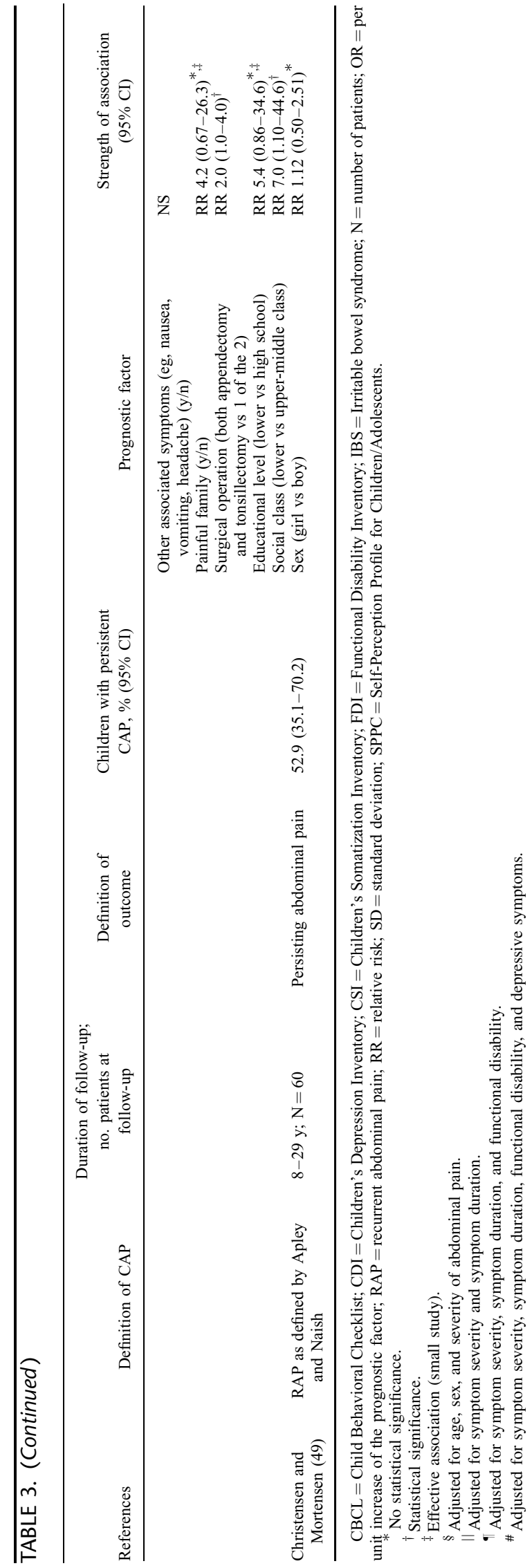

\section{DISCUSSION}

In this systematic review, we investigated and summarized the quantity and quality of all of the current evidence for potential prognostic factors for persistence of CAP in children. In total, 8 prospective cohort studies were included, of which 5 were at low risk for bias. Seventeen potential prognostic factors for the persistence of CAP were identified, of which 10 were examined in 1 study only. Consequently, beforehand, the level of evidence for an association between these factors and the persistence of CAP was low.

We found moderate evidence that having a parent with functional GI symptoms predicted the persistence of CAP in children, and weak evidence that parental perception of illness predicted the persistence of CAP. These findings can be explained by the social learning theory of illness behavior (50). Illness behavior is the way a person reacts to changes in health conditions. People with an inappropriate illness behavior misconceive normal sensations as symptoms of disease and react to stressful events with somatic symptoms such as abdominal pain. Children tend to copy their parents' behavior, and parents subsequently reinforce this heightened illness behavior by paying positive attention to the complaints. Consistent with this theory is the finding that cognitive-behavioral family interventions can successfully reduce the child's pain (51). In this therapy, the pain process is thoroughly explained and parents are thought to reinforce well behavior and distract the child during pain episodes (52). Heredity may also play a role; CAP and irritable bowel syndrome can run in families, and genetic factors may contribute to the association of parent and child symptoms (53).

We found strong evidence that female sex had no predictive value for the persistence of CAP. This was a consistent finding in 5 of 6 studies in the present review and is also supported by others (54). Two prospective cohort studies, however, reported that girls in comparison to boys had an increased risk to develop CAP $(18,19)$. Thus, the association between sex, incidence, and persistence of CAP is not yet fully elucidated and requires additional study. The severity of abdominal pain was not a risk factor for the persistence of CAP in children; this finding applied to all of the included studies. In addition, we found weak evidence that negative life events predicted the persistence of CAP, which is in accordance with current opinion.

In contrast to current opinion (10), we did not find evidence for an association between psychological factors of the child and the persistence of CAP. On the contrary, we found conflicting evidence as to whether psychological factors of the child "prevented" or had no predictive value for the persistence of CAP in children. One possible explanation for this finding is that CAP and psychological factors may be triggered by the same underlying mechanisms and occur together, but are not necessarily causally related. Therefore, although children with CAP may be at risk for psychological disorders, the psychological disorders themselves are not the reason for the persistence of abdominal pain. Another explanation for the lack of observed evidence is the small number of studies and the risk of bias in those studies that investigated the prognostic value of psychological factors. An argument for a relation between children's psychological problems and persistence of CAP is that psychological treatments reduce abdominal pain (51). Thus, in the included studies, children with psychological problems who were less likely to have persistent CAP may have received psychological treatments; however, the authors of the individual studies did not report on the treatment used.

\section{Limitations}

The following limitations should be taken into account when interpreting the results of the present review. First, some studies 
TABLE 4. Best-evidence synthesis

No. studies with their risk for bias and direction

Total no. of association with persistent

Prognostic factors studies

CAP at follow-up

Best-evidence synthesis

Patient characteristics

Female sex

Young age

Low educational level of child

Psychological factors of child

Behavioral disturbances

Psychological disorders

Depressive or anxiety disorders

Self-perceived academic competence

"Any" psychological factor

$(2.1+2.2+2.3+2.4)$

Pain history and associated symptoms at baseline

Long duration of abdominal pain

Severe abdominal pain

History of $\geq 2$ surgical operations

Presence of other associated symptoms such as nausea, vomiting, and headaches

High levels of functional disability

Environmental factors

Low SES

High levels of negative life events

Family factors

High levels of functional GI complaints in family

Illness perception

Attitude toward health care
6

4

4 LRB: 3 NA $(42,44,46)$, $1+(43) ; 2$ HRB:

2 NA $(48,49)$

3 LRB: 2 NA $(42,44)$, $1+(45) ; 1$ HRB:

NA (48)

1 HRB: + (48)

1 LRB: NA (43)

1 LRB: NA (44)

1 LRB: - (45);

1 HRB: + (47)

1 HRB: + (47)

3 LRB: 2 NA $(43,44)$, 1- (45); 1 HRB: + (47)

2 LRB: + (45), NA (43);

2 HRB: 2 NA $(47,48)$

1 LRB: NA (44); 2 HRB:

2 NA $(47,48)$

1 HRB: + (48)

1 HRB: NA (48)

1 HRB: + (47)

1 HRB: + (48)

1 LRB: + (44)

2

1 LRB: mother's + (45);

1 HRB: parent's + (48)

1

1 LRB: a high number of involved consultants + (44) lack of insight into psychological influences on pain $+(44)$, disagreement with psychological referral $+(44)$

1 LRB: parental manipulative complaining + (44), complaining through hospital system NA (44), hostility toward health workers NA (44)
Strong evidence for no predictive value

Conflicting

Insufficient

Weak evidence for no predictive value

Weak evidence for no predictive value

Conflicting

Insufficient

Conflicting evidence for no predictive value or a negative predictive value

Conflicting

Moderate evidence for no predictive value

Insufficient

Insufficient

Insufficient

Insufficient

Weak evidence for a positive predictive value

Moderate evidence for a positive predictive value

Weak evidence for a positive predictive value

Conflicting 
may have been missed during the literature search because CAP is a description of symptoms and there is no single, commonly used name or definition for it. However, the chance that studies are missed is small because a sensitive search strategy was used that included all of the current names and definitions used for CAP. Second, because all of the studies included in the present review were conducted in tertiary or secondary care centers, the results of these studies are not representative for a community setting or general practice. In addition, it is important to note that the prognosis of CAP is influenced by many factors and that these may interact and increase/decrease each other's effect. Most of the studies in the present review used univariate analysis and did not take possible interactions into consideration.

There is no internationally accepted method to conduct systematic reviews of prognostic studies. In addition, there are no validated or widely used criteria to assess the risk of bias. Hayden et al (15) and the STROBE statement (16) have provided some recommendations for the methodological assessment of reviews, and we incorporated them in our analysis of bias. Although not recommended, we used an overall score for risk for bias to enable a best-evidence synthesis.

In the present review, strict inclusion criteria were applied. Only CAP, as defined by Apley and Naish (2), von Baeyer and Walker (4), or the Rome committee (3), was eligible for inclusion. In our opinion, the inclusion of studies using different criteria for CAP did not make our study population heterogeneous. All of the studies defined CAP as an abdominal pain of comparable duration and frequency, and all of the studies excluded children with organic pathology. Therefore, the results of this review are applicable to referred children with CAP in whom organic pathology has been ruled out by additional diagnostic testing. Another consequence of our inclusion criteria is that some studies on CAP were not included. Ramchandani et al (23) described the 1-year outcome of 860 children with RAP. The latter study was not included because the outcome measure was abdominal pain in the previous year (a "yes/no" question). In the definition provided by the authors, the rate of abdominal pain is not comparable with the rate of abdominal pain in our definition; we doubt whether their reported abdominal pain could be regarded as CAP.

\section{Additional Research}

The present study clearly shows the value of a systematic review because it demonstrates that there is little evidence to support all of the current assumptions on pediatric CAP. This emphasizes the need for well-conducted follow-up studies on pediatric CAP, not only in referred children but also in primary care and open populations. The outcome measure should be well defined and, besides the persistence of abdominal pain, should include functional disability due to abdominal pain. The criterion to rule out organic causes should not be part of the definition of outcome because discussion will always remain as to how much and what kind of medical investigation is needed to be sure of ruling out an organic basis for the abdominal pain. Including this criterion will make the definition impossible to apply, for example, to a community or school population. Multivariable analyses are needed to reveal possible interactions between potential prognostic factors. Such interactions can have important clinical consequences. For example, whereas it is impossible to prevent a child from having negative life events, it may be possible to strengthen factors that can diminish their effects.

\section{Clinical Implications}

Boys and girls were found to be at similar risks for persistence of CAP. This implies that the clinician should be aware that in both girls and boys, CAP may persist. Furthermore, our results indicate that the clinician should be aware of the association between parental GI symptoms, parental perception toward the abdominal pain, and the persistence of abdominal pain in the child. Adequate follow-up should be part of the management of a child with functional abdominal pain at increased risk for chronicity. By addressing coping strategies and parental cognitions on abdominal pain, the persistence of abdominal pain may be reduced or prevented. Last, our finding that the severity of abdominal pain was not a risk factor for the persistence of CAP in children may be particularly useful for clinicians in their management of pediatric CAP. A child in severe pain is impressive and may therefore lead clinical management. The finding of this review may help to change this behavior.

\section{CONCLUSIONS}

Children with parents with GI symptoms are at risk for the persistence of CAP. Female sex and the severity of CAP do not influence the persistence of pediatric CAP. The current opinion that a child's psychological disorders predict the persistence of CAP is not supported by evidence from prognostic studies. Our results are based on a limited amount of studies and should therefore be interpreted with caution.

\section{REFERENCES}

1. Chitkara DK, Rawat DJ, Talley NJ. The epidemiology of childhood recurrent abdominal pain in Western countries: a systematic review. Am J Gastroenterol 2005;100:1868-75.

2. Apley J, Naish N. Recurrent abdominal pains: a field survey of 1,000 school children. Arch Dis Child 1958;33:165-70.

3. Rasquin-Weber A, Hyman PE, Cucchiara S, et al. Childhood functional gastrointestinal disorders. Gut 1999;45 (Suppl 2):1160-8.

4. von Baeyer CL, Walker LS. Children with recurrent abdominal pain: issues in the selection and description of research participants. $J$ Dev Behav Pediatr 1999;20:307-13.

5. Jones MP, Dilley JB, Drossman D, et al. Brain-gut connections in functional GI disorders: anatomic and physiologic relationships. Neurogastroenterol Motil 2006;18:91-103.

6. Scharff L. Recurrent abdominal pain in children: a review of psychological factors and treatment. Clin Psychol Rev 1997;17:145-66.

7. Campo JV, Bridge J, Lucas A, et al. Physical and emotional health of mothers of youth with functional abdominal pain. Arch Pediatr Adolesc Med 2007;161:131-7.

8. Gieteling M, Bierma-Zeinstra S, Passchier J, et al. Prognosis of chronic or recurrent abdominal pain in children. J Pediatr Gastroenterol Nutr 2008:47:316-26.

9. Chitkara DK, van Tilburg MA, Blois-Martin N, et al. Early life risk factors that contribute to irritable bowel syndrome in adults: a systematic review. Am J Gastroenterol 2008;103:765-74.

10. Levy RL, Olden KW, Naliboff BD, et al. Psychosocial aspects of the functional gastrointestinal disorders. Gastroenterology 2006;130:144758.

11. Altman DG. Systematic reviews of evaluations of prognostic variables. BMJ 2001;323:224-8.

12. Wilczynski NL, Haynes RB. Optimal search strategies for detecting clinically sound prognostic studies in EMBASE: an analytic survey. J Am Med Inform Assoc 2005;12:481-5.

13. Borghouts JA, Koes BW, Bouter LM. The clinical course and prognostic factors of non-specific neck pain: a systematic review. Pain 1998;77:113.

14. Hudak PL, Cole DC, Frank JW. Perspectives on prognosis of soft tissue musculoskeletal disorders. Int J Rehabil Res 1998;21:29-40.

15. Hayden JA, Cote P, Bombardier C. Evaluation of the quality of prognosis studies in systematic reviews. Ann Intern Med 2006;144:427-37.

16. von Elm E, Altman DG, Egger M, et al. The Strengthening the Reporting of Observational Studies in Epidemiology (STROBE) statement: guidelines for reporting observational studies. Ann Intern Med 2007;147:573-7. 
17. Sackett DL, Straus SE, Richardson WS. Evidence-based Medicine. How to Practice and Teach EBM. Guidelines. Edinburgh: Churchill Livingstone; 2000.

18. Ramchandani PG, Stein A, Hotopf M, et al. Early parental and child predictors of recurrent abdominal pain at school age: results of a large population-based study. J Am Acad Child Adolesc Psychiatry 2006; 45:729-36.

19. El-Metwally A, Halder S, Thompson D, et al. Predictors of abdominal pain in schoolchildren: a 4-year population-based prospective study. Arch Dis Child 2007;92:1094-8.

20. Wasserman AL, Whitington PF, Rivara FP. Psychogenic basis for abdominal pain in children and adolescents. J Am Acad Child Adolesc Psychiatry 1988;27:179-84.

21. Hyams JS, Davis P, Sylvester FA, et al. Dyspepsia in children and adolescents: a prospective study. J Pediatr Gastroenterol Nutr 2000; 30:413-8.

22. Kolts RL, Nelson RS, Park R, et al. Exploratory laparoscopy for recurrent right lower quadrant pain in a pediatric population. Pediatr Surg Int 2006;22:247-9.

23. Ramchandani PG, Fazel M, Stein A, et al. The impact of recurrent abdominal pain: predictors of outcome in a large population cohort. Acta Paediatr 2007:96:697-701.

24. Walker LS, Greene JW. Negative life events and symptom resolution in pediatric abdominal pain patients. J Pediatr Psychol 1991;16:341-60.

25. Walker LS, Garber J, Greene JW. Somatic complaints in pediatric patients: a prospective study of the role of negative life events, child social and academic competence, and parental somatic symptoms. J Consult Clin Psychol 1994;62:1213-21.

26. Walker LS, Garber J, Van Slyke DA, et al. Long-term health outcomes in patients with recurrent abdominal pain. J Pediatr Psychol 1995;20:23345.

27. Gremse DA, Nguyenduc GH, Sacks AI, et al. Irritable bowel syndrome and lactose maldigestion in recurrent abdominal pain in childhood. South Med J 1999;92:778-81.

28. Claar RL, Walker LS, Smith CA. Functional disability in adolescents and young adults with symptoms of irritable bowel syndrome: the role of academic, social, and athletic competence. J Pediatr Psychol 1999; 24:271-80.

29. Walker LS, Claar RL, Garber J. Social consequences of children's pain: when do they encourage symptom maintenance? J Pediatr Psychol 2002;27:689-98.

30. Walker LS, Smith CA, Garber J, et al. Testing a model of pain appraisal and coping in children with chronic abdominal pain. Health Psychol 2005;24:364-74.

31. Mulvaney S, Lambert EW, Garber J, et al. Trajectories of symptoms and impairment for pediatric patients with functional abdominal pain: a 5-year longitudinal study. J Am Acad Child Adolesc Psychiatry 2006;45: $737-44$.

32. Dahl L, Haahr J. Recurrent abdominal pain in children. Ugeskr Laeger 1969;131:1509-12.

33. Apley J, Hale B. Children with recurrent abdominal pain: how do they grow up? BMJ 1973;3:7-9.

34. Liebman WM. Recurrent abdominal pain in children: a retrospective survey of 119 patients. Clin Pediatr (Phila) 1978;17:149-53.

35. Stickler GB, Murphy DB. Recurrent abdominal pain. Am J Dis Child 1979;133:486-9.
36. Bury RG. A study of 111 children with recurrent abdominal pain. Aust Pediatr J 1987;23:117-9.

37. Oymar K, Fluge G, Rosendahl K. Recurrent abdominal pain. A prospective study of 68 children. Tidsskr Nor Laegeforen 1993;113:2566-8.

38. Borge AI, Nordhagen R, Moe B, et al. Prevalence and persistence of stomach ache and headache among children. Follow-up of a cohort of Norwegian children from 4 to 10 years of age. Acta Paediatr 1994; 83:433-7.

39. Hotopf M, Carr S, Mayou R, et al. Why do children have chronic abdominal pain, and what happens to them when they grow up? Population based cohort study. BMJ 1998;316:1196-200.

40. Croffie JM, Fitzgerald JF, Chong SK. Recurrent abdominal pain in children-a retrospective study of outcome in a group referred to a pediatric gastroenterology practice. Clin Pediatr (Phila) 2000;39:26774.

41. Campo JV, Di Lorenzo C, Chiappetta L, et al. Adult outcomes of pediatric recurrent abdominal pain: do they just grow out of it? Pediatrics 2001;108:E1.

42. Pace F, Zuin G, Di Giacomo S, et al. Family history of irritable bowel syndrome is the major determinant of persistent abdominal complaints in young adults with a history of pediatric recurrent abdominal pain. World J Gastroenterol 2006;12:3874-7.

43. Stordal K, Nygaard EA, Bentsen BS. Recurrent abdominal pain: a fiveyear follow-up study. Acta Paediatr 2005;94:234-6.

44. Lindley KJ, Glaser D, Milla PJ. Consumerism in healthcare can be detrimental to child health: lessons from children with functional abdominal pain. Arch Dis Child 2005;90:335-7.

45. Crushell E, Rowland M, Doherty M, et al. Importance of parental conceptual model of illness in severe recurrent abdominal pain. Pediatrics 2003;112:1368-72.

46. Walker LS, Guite JW, Duke M, et al. Recurrent abdominal pain: a potential precursor of irritable bowel syndrome in adolescents and young adults. J Pediatr 1998;132:1010-5.

47. Walker LS, Heflinger CA. Quality of life predictors of outcome in pediatric abdominal pain patients: Findings at initial assessment and 5-year follow-up. In: Drotar D, ed. Measuring Health Related Quality of Life in Children and Adolescents: Implications for Research and Practice. Mahwah, NJ: Lawrence Eribaum; 1998:237-52.

48. Magni G, Pierri M, Donzelli F. Recurrent abdominal pain in children: a long term follow-up. Eur J Pediatr 1987;146:72-4.

49. Christensen MF, Mortensen O. Long-term prognosis in children with recurrent abdominal pain. Arch Dis Child 1975;50:110-4.

50. Levy RL, Langer SL, Whitehead WE. Social learning contributions to the etiology and treatment of functional abdominal pain and inflammatory bowel disease in children and adults. World J Gastroenterol 2007;13:2397-403.

51. Huertas-Ceballos A, Logan S, Bennett C, et al. Psychosocial interventions for recurrent abdominal pain (RAP) and irritable bowel syndrome (IBS) in childhood. Cochrane Database Syst Rev 2008;23:1:CD003014.

52. Walker LS, Williams SE, Smith CA, et al. Parent attention versus distraction: impact on symptom complaints by children with and without chronic functional abdominal pain. Pain 2006;122:43-52.

53. Saito YA, Talley NJ. Genetics of irritable bowel syndrome. Am $J$ Gastroenterol 2008;103:2100-4.

54. Berger MY, Gieteling MJ, Benninga MA. Chronic abdominal pain in children. BMJ 2007;334:997-1002. 